BNL- 64531.

CAP-190-ATF-97C

INVERSE FREE ELECTRON LASER ACCELERATION WITH A SQUARE WAVE WIGGLER

\author{
Z. Parsa \\ Department of Physics \\ Brookhaven National Laboratory \\ Upton, NY 11973 \\ and \\ M.P. Pato \\ Instituto de Fisica, Univ. de Sao Paulo \\ c.P. 20516, 01498 Sao Paulo, S.P., Brazil
}

June 1997 


\title{
Inverse Free Electron Laser Acceleration with a Square Wave Wiggler
}

\author{
Z. Parsa ${ }^{\dagger 1}$ and M.P. Pato ${ }^{\ddagger 2}$ \\ $\dagger$ Brookhaven National Laboratory \\ 901A Physics Dept., Upton, NY 111973-5000, USA

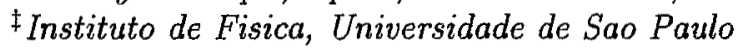 \\ C.P.20516,01498 Sao Paulo,S.P., Brazil
}

\begin{abstract}
We present an Inverse Free Electron Laser with a Square Wave Wiggler (IFELSW) as a new acceleration scheme and show Analytically and numerically about factor of 2 gain in the energy when compared to the standard IFEL with the Sinusoidal [1] field Wiggler.
\end{abstract}

\section{INTRODUCTION}

The Nonlinear Amplification of Inverse-Bremsstrahlung Electron Acceleration (NAIBEA) is a scheme of acelerating charged particles that uses a laser coupled to a static applied field structure in which a constant magnetic, or electric, field alternates sign at some appropriately determined positions in such a way that the particle is always accelerated. This may be understood as a kind of the Inverse Free Electron Laser Acceleration (IFEL). In both of these acceleration schemes [1,2], relativistic particles move under the combined action of an electromagnetic travelling wave, the laser field, and a magnetic, or electric, applied static field. Both, the laser radiation and the beam of particles, propagate along the field structure. This static field, usually provided by magnets, acts like a wiggler by producing a small undulation in the particle trajectory. The transverse velocity of this undulation couples in such a way to the electric field of the electromagnetic wave that the energy is transferred from the laser to the beam.

In the standard IFEL this wiggling motion is created by an undulator whose magnetic field varies sinusoidally with the longitudinal distance [1], while in

1) Supported by US Department of Energy Contract No. DE-AC02-76CH00016 and National Science Foundation NSF-PHY-94-071.94

2) Supported in part by the CNPq-Brazil 


$$
\begin{gathered}
\frac{d P_{z}}{d t}=-e \beta_{x} B_{y}-e \beta_{x} B_{a p p} \\
\frac{d P_{x}}{d t}=-e E_{x}+e \beta_{z} B_{y}+e \beta_{z} B_{a p p} .
\end{gathered}
$$

With $E_{x}=B_{y}=-E_{x_{0}} \sin \phi, \phi=k(c t-z)$ and $B_{a p p}=B_{0} f(\phi)$, where $\phi$ corresponds to the phase of the laser field at the particle position, the above equations become

$$
\begin{gathered}
\frac{d P_{z}}{d t}=e \beta_{x} E_{x_{0}} \sin \phi-e \beta_{x} B_{0} f(\phi) \\
\frac{d P_{x}}{d t}=e\left(1-\beta_{z}\right) E_{x_{0}} \sin \phi+e \beta_{z} B_{0} f(\phi)
\end{gathered}
$$

where $f(\phi)$ is a unit square wave of period $2 \pi$ that switch signs at odd numbers of $\frac{\pi}{2}$ and $f(0)=-1$. By multiplying the above equations by $v_{z}$ and $v_{x}$, respectively, we obtain the energy equation

$$
m c^{2} \frac{d \gamma}{d t}=e v_{x} E_{x_{0}} \sin \phi
$$

Eqs. (4) and (6) can be combined to give

$$
c \frac{d P_{z}}{d t}-m c^{2} \frac{d \gamma}{d t}=-e v_{x} B_{0} f(\phi)
$$

First integrals of the motion are obtained by integrating Eqs. (4) and (5)

$$
\begin{gathered}
P_{z} c=m c^{2} \gamma-e \int_{0}^{t} d t v_{x} B_{0} f(\phi)+K_{1} \\
P_{x}=-e E_{x_{0}} \cos \phi-e \int_{0}^{t} d t v_{z} B_{0} f(\phi)+K_{2}
\end{gathered}
$$

where we used the fact that $\frac{d \phi}{d t}=k c\left(1-\beta_{z}\right)$. These two integrals can be performed by parts using the fact that since $f(\phi)$ is a square wave function its derivative is a sum of delta functions, namely,

$$
f^{\prime}(\phi)=2 \sum_{n}(-1)^{n} \delta\left[\phi-(2 n+1) \frac{\pi}{2}\right] .
$$

We obtain

$$
P_{z} c=m c^{2} \gamma-e B_{0} x(\phi) f(\phi)+K_{i}
$$




$$
P_{x}=-e E_{x_{0}} \cos \phi-e B_{0} z(\phi) f(\phi)-2 e B_{0} \sum_{n=1}^{i}(-1)^{n} z_{n}+K_{2}
$$

where

$$
K_{i}=-K_{1}-e B_{0} x_{0}+2 B_{0} \sum_{n}^{i}(-1)^{n} x_{n}
$$

and $i$ is the running index corresponding to the segment of the wiggler where the particle is located.

In reducing the above system of equations to a one dimensional problem, for convenience we first change the independent variable from the time $(\mathrm{t})$ to $(\phi)$, and use a new variable $Q$ such that $\frac{d Q}{d \phi}=P_{x}$, i.e.,

$$
Q^{\prime}=P_{x}=m \gamma \frac{d \phi}{d t} x^{\prime}(\phi)=\frac{k}{c}\left(m c^{2} \gamma-P_{z}\right) x^{\prime}(\phi)
$$

Where the primes denote derivatives with respect to $\phi$ and we have used the relation $\frac{d \phi}{d t}=k\left(c-v_{z}\right)=\frac{k}{m c \gamma}\left(m c^{2} \gamma-P_{z}\right)$. Substituting $P_{z}$ in Eq. 14 results in

$$
Q^{\prime}=-\frac{k}{c} B_{0} x(\phi) x^{\prime}(\phi) f(\phi)-\frac{k}{c} K_{i} x^{\prime}(\phi) .
$$

and after integrating we obtain

$$
Q=-\frac{k}{2 c} e B_{0} x^{2}(\phi) f(\phi)-\frac{k}{c} K_{i} x(\phi)+C_{i}
$$

where the constant $C_{i}$ is introduced in order to assure the continuity of coordinate $Q$ when the particle traverses the switching sign points of the field: They are given by

$$
C_{i}=\frac{e B_{0}}{k c} \sum_{n}^{i}(-1)^{n}\left(k x_{n}\right)^{2}+\frac{K_{i} k x_{0}}{c}
$$

Since $Q^{\prime \prime}=P_{x}^{\prime}$, Eq. (9) and relativistic relations can be used to eliminate the dependence of $Q$ in other variables except $\phi$ (the phase of the laser field). This leads to the following equation of motion for $Q$ as a function of $\phi$ only:

$$
Q^{\prime \prime}=-\frac{e B_{0} c^{2}}{2} f(\phi) \frac{m^{2} c^{2}+Q^{2}}{k K_{i}^{2}-2 c e B_{0} f(\phi)\left(Q-C_{i}\right.}+e B_{a p p} f(\phi)+e E_{x_{0}} \sin \phi
$$

This is a nonlinear second order differential equation. It can be used to predict the existence of a periodic solution as illustrated In Fig.3, We have the evolution of the variable $Q$ and of the transversal displacement $x$, obtained inverting Eq. (16), as a function of $\phi$. This is a resonant trajectory. It is 
locally periodic, in the sense that its amplitude and period vary slowly as the particle accelerates.

We observe in Fig. 3 that the electron enters and leaves each segment (in which the applied field has a given sign), with $x=0$ which means that the constants $K_{i}$ and $C_{i}$ (given by Eqs. (13) and (17)) remain the same in all segments. We further note, that multiplying the equation (18) by $f(\phi)$ the product $f(\phi) Q(\phi)$ satisfies an equation which remains invariant, since $f^{2}(\phi)=1$ and $f(\phi) \sin \phi$ does not change from one segment to the other. As a consequence an oscilatory periodic motion for $Q$ follows. The relatively small perturbation to the periodicity being caused by the lack of symmetry of the laser field term (the last term in Eq.18) inside each segment of the square wave.

A solution to this nonlinear second order differential equation can be constructed and used to obtain an expression for the rate of energy increase. However here, we follow a simpler approximate approach by obtaining from Eq. (12), an expression for the transversal velocity $v_{x}$ and puting it into the Eq. (6) (neglecting the oscilatory parts, as we are not interested in). We then observe that in a given segment of the applied field, the variation of the longitudinal distance $z$ is exactly half of the wiggler period $\Lambda_{w}$. Since $z$ is a monotonic function of $\phi$, (in the average) we can further assume that $z$ increases linearly with $\phi$. Then the two terms left in the expression of $v_{x}$ define a triangular wave. So we can write,

$$
v_{x} \sim \frac{e B_{0} \Lambda_{w}}{4 m c^{2} \gamma} g(\phi)
$$

where $g(\phi)$ is a unit triangular wave with the Fourier representation

$$
g(\phi)=-\sum_{n=1}^{\infty} \frac{8}{n^{2} \pi^{2}} \sin \frac{n \pi}{2} \sin n \phi
$$

Substituting this into Eq.19 and equation 19 into Eq.6 (again neglecting the high frequency terms) we find,

$$
\frac{d \gamma}{d t}=\frac{e E_{x_{0}}}{m c^{2}} \frac{e B_{0} \Lambda_{w}}{8 \gamma m c^{2}}
$$

This expression is to be compared (as in e.g. [10]) with the equivalent one for a sinusoidal wiggler and a plane polarized wave (Eq. (27) of Ref. [1]). We find that the square wiggler gives a rate of increase that is about two times larger.

\section{CONCLUSION}

We found in the last section, that it is possible to construct an Inverse Free Electron Laser (IFEL) with a square wave wiggler (IFELSW) such that 
the particle in a resonant trajectory is always accelerated. The particles are supposed to be injected along with the laser radiation, i.e., with no transversal velocity. By giving to the square wave a phase shift of $\frac{\pi}{2}$ the wiggling motion acquired by the electrons will be in phase with the laser field and they will be accelerated.

It was possible to obtain an explicit expression for the average rate of energy increase and as a result we have found a gain in energy of about two times when compared with the standard IFEL with a sinusoidal wiggler. We note that, although Maxwell's equations do not allow an abrupt change of sign of the field, the results we have obtained here suggest that the acceleration scheme using laser field turns out to be more efficient as we approach the square wave field patterns.

\section{REFERENCES}

1. E.D. Courant, C. Pellegrini and W. Zakowicz, Phys.Rev. A32, 2813 (1985).

2. M.S. Hussein and M.P. Pato, Phys. Rev. Lett. 68, 1136 (1992).

3. M.P. Pato, M.S. Hussein, and A.K. Kerman, Nucl. Inst. \& Meth. in Phys. Res.A328, 342 (1993).

4. M.S. Hussein, M.P. Pato and A.K. Kerman, Phys. Rev. A46, 11363562 (1992).

5. M.S. Hussein, M.P. Pato, Mod. Phys. Lett. B6, 747 (1992).

6. M.S. Hussein and M.P. Pato, Int. Jour. of Mod. Phys. A8, 3235 (1992).

7. R.M.O. Galvão, M.S. Hussein, M.P. Pato, and A. Serbeto, Phys.Rev. E49, R4807 (1994).

8. M. P. Pato, Private communication.

9. Z. Parsa, 'An Inverse Free Electron Laser with a Square Wave Wiggler', Propsal to ATF Steering Committee, Report - October 30, 1996.

10. Z. Parsa, 'Square Wave Wiggler IFEL' Supporting Information for ATF Steering Committee Review Meeting, Report - November 20, 1996; and references therein (1996);

11. Z. Parsa, 'Inverse Free Electron Laser Acceleration Scheme with a Square Wave Wiggler', Presentation UCSB/ITP-96, Santa Barbara, CA (1996).

12. Z. Parsa 'New IFEL' Experimental Proposal for BNL-ATF (1997).

13. Z. Parsa, 'Improved NAIBEA and IFEL', APS-APR97 No. 5933, Presentation Washington D.C. (1997).

14. Z. Parsa, T. Marshall, 'Enhanced IFEL Experiment using a Novel Wiggler' PAC97 Presentation, Vancouver, Canada (May 1997).

\section{Figure Captions:}

Fig.1- Show the directions of the laser field and Injection of Electrons.

Fig.2- A sketch of the Wiggler Magnetic field.

Fig.3- Show the trajectories as the electron moves through the Wiggler.

Fig.4- The evolution of energy as the electron moves through the Wiggler.

Fig.5- Energy distribution in terms of $\gamma$. 


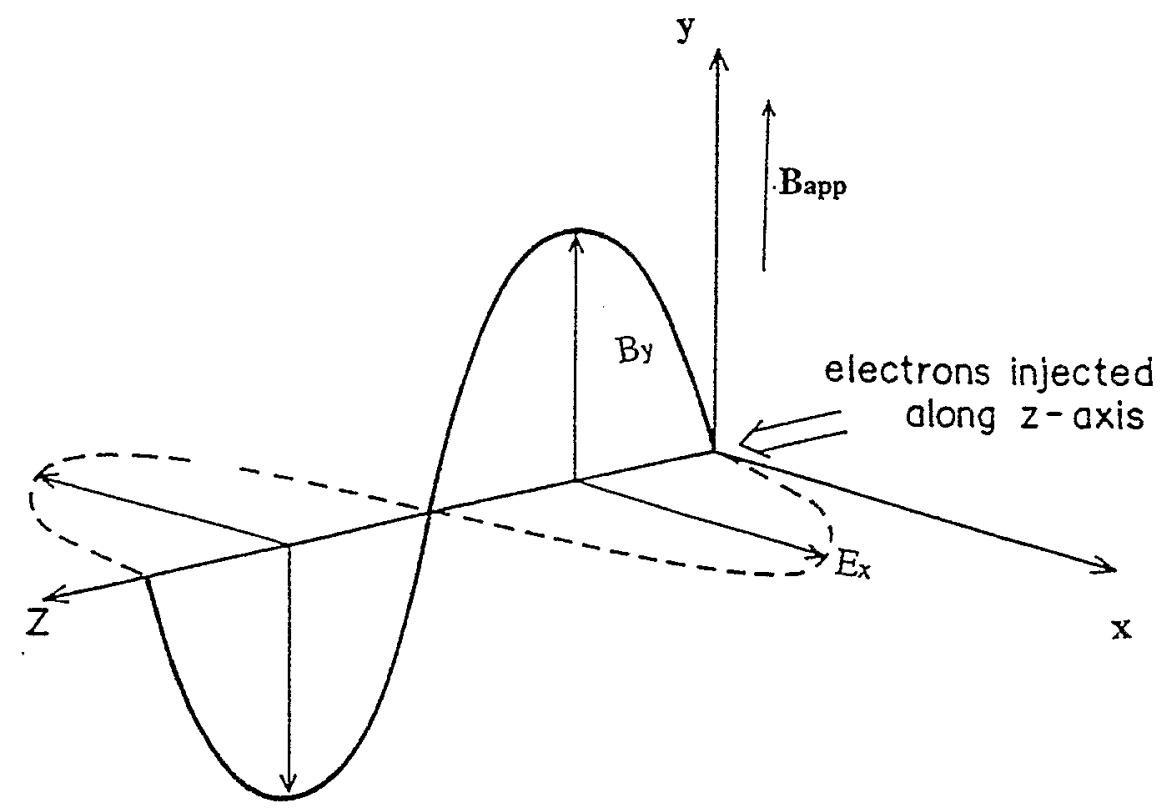

Fig.1-.: Plot of the directions of the laser field and injection of electrons. 


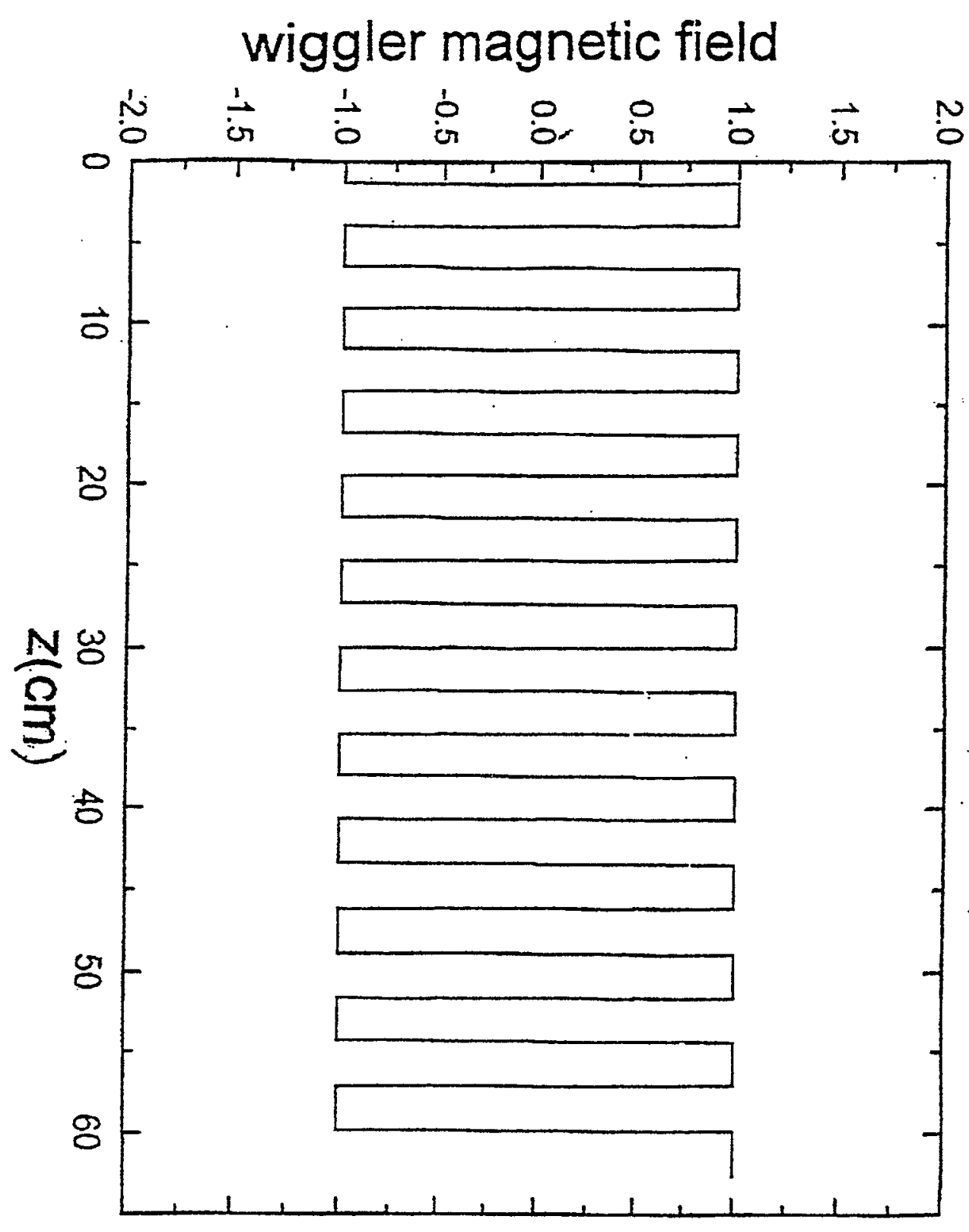

Fig.2- .: A sketch of the Wiggler Magnetic field for the proposed IFELSW and the positions where the sign of the magnetic field is to be switched 


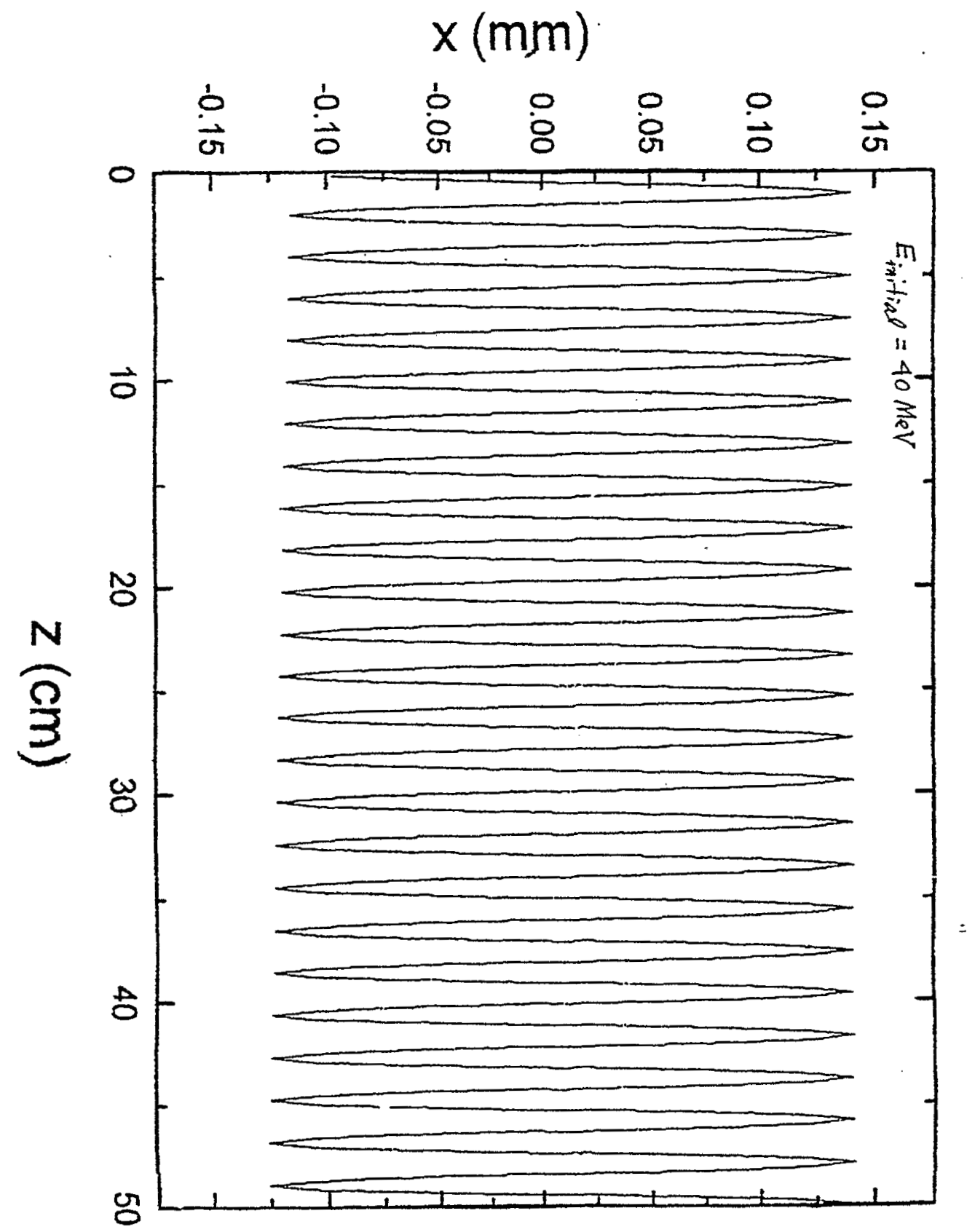

Fig.3- Plot of the trajectories as the electron moves through the Wiggler (case a Ref. [9]) 


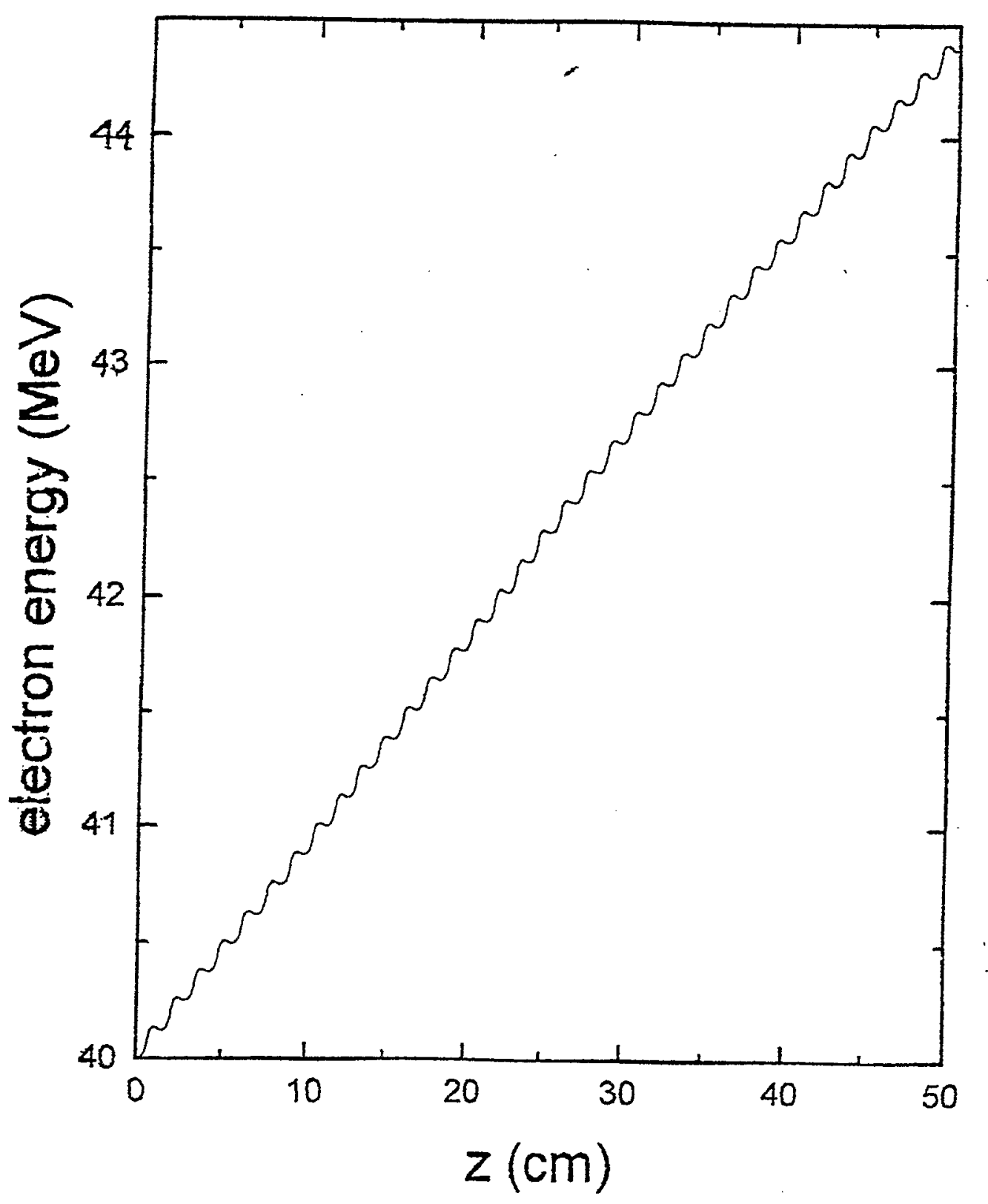

Fig.4-: Plot showing the predicted IFELSW evolution of the energy as the electron moves through the Wiggler (case $a$, where the initial prameters are the same as recent BNL- ATF IFEL experiment (case a Ref. [9]) 

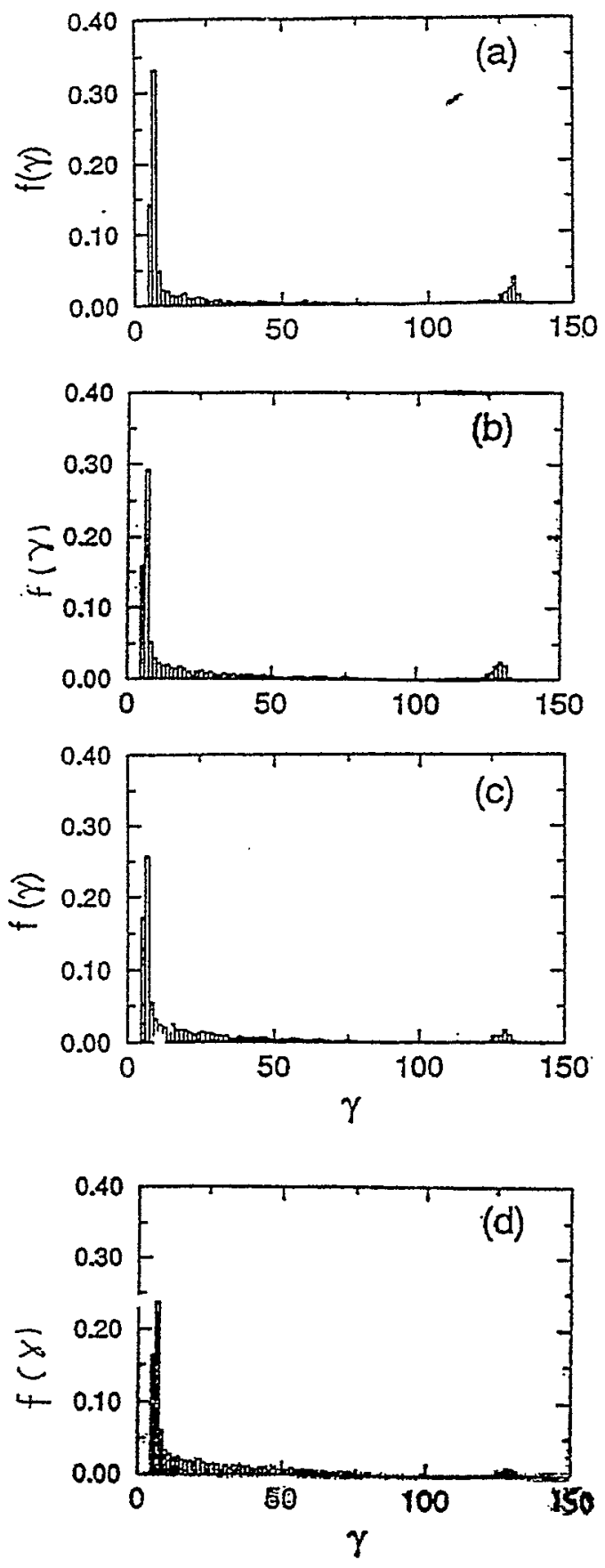

Fig.5- ; Plot showing Energy distribution in terms of $\gamma$ as a normalized histogram for different Temperatures of $10-150 \mathrm{KeV}$ for example of the initial density of $10^{* *} 8 \mathrm{~cm}^{* *}-3$. Other simulations can be done.

a) $\mathrm{T}=10 \mathrm{KeV}$, b) $\mathrm{T}=50 \mathrm{KeV}$, c) $\mathrm{T}=100 \mathrm{KeV}$, d) $\mathrm{T}=150 \mathrm{KeV}$. 